\title{
A note on memory for sense: Incidental recognition of warnings phrased as conditionals, disjunctives, and conjunctives
}

\author{
SAMUEL FILLENBAUM \\ University of North Carolina, Chapel Hill, North Carolina 27514
}

\begin{abstract}
Warnings may be phrased as conditionals, as conjunctive sentences, or as disjunctive sentences with the first clause negated; and subjects judge warnings variously phrased as above to the equivalent in meaning. Performance on an incidental recognition test after a cover rating task indicates that there is much confusion among the different phrasings of warnings even when these involve such radically different sentence operators as IF, AND, and OR and suggests that memory is responsive to sense or meaning rather than the particular form in which it is realized.
\end{abstract}

There is by now considerable evidence to suggest that, characteristically, it is not the surface form nor even the deep structure of sentences that is remembered, but rather semantic information or propositional content (for a survey of relevant work, see Fillenbaum, 1973). The study described below will present some further data to this point for a case involving a class of sentences using one or the other of the three logical particles-AND, OR, and IF. This evidence is of interest not only because it provides additional support for the general thesis that memory is for sense, but also because it does so in a context involving three basic well-nigh indispensable sentence operators.

Warnings can be phrased more or less equivalently as conditionals ("If you drop the glass, it will break."), as conjunctives ("Drop the glass and it will break."), or as disjunctives with the first clause negated ("Don't drop the glass or it will break."). Indeed when subjects were given sentence pairs involving warnings where the first sentence was always a conditional (if $p, q$ ) and the second sentence either (a) a conjunctive ( $p$ and $q$ ), or (b) a disjunctive with first clause negated (not $p$ or $q$ ), the sentence pairs were judged to be equivalent in meaning $94 \%$ and $98 \%$ of the time, respectively (while this was true only $16 \%$ of the time if the second sentence in the pair was a disjunctive of the form " $p$ or not q"); see Fillenbaum (Note 1). If warnings variously phrased as conditionals, as conjunctives, or as certain sorts of disjunctives are indeed equivalent in meaning, then one might expect considerable memorial confusion for such different phrasings, especially if subjects did not expect their memory to be tested, e.g., if an incidental recognition task is employed.

This study was supported in part by U. S. Public Health Service Grant 10006 from the National Institute of Mental Health.

\section{METHOD}

Subjects were asked to rate each of a series of sentences as normal/ordinary or extraordinary/strange; and immediately afterward, quite unexpectedly for them, they were given a recognition test on the sentences. The sentences were all warnings, either (a) more or less normal, or (b) perverse in that the normal or expected relation between the two propositions was negated, e.g., "Stay out in the sun too long and you won't get burned." On the original rating task, each sentence was in one of three forms which may be symbolized as: (a) if p, q; (b) $p$ and $\mathrm{q}$; and (c) not $\mathrm{p}$ or $\mathrm{q}$. Given two kinds of sentences, normal and perverse warnings, there were thus six sentence sets (with six items in each set). On the recognition test, a sentence could occur in any one of six forms. Consider, for example, a normal warning originally phrased as an IF sentence. It could appear (1) verbatim, as an IF sentence again; or, (2) and (3) as its mate in AND and OR, respectively; or, (4), (5), and (6), changed into a perverse warning in IF, AND, and OR, respectively. Thus, on the recognition task, phrasing (in IF, AND, and OR) and whether the sentence maintained or changed its conceptual form, as normal or perverse, was simultaneously manipulated. There were thus 36 possibilities with one item for each, plus a few fillers at the start and end, making for a total of 43 items. There were 62 subjects in the experiment, undergraduates fulfilling a course requirement.

\section{RESULTS AND DISCUSSION}

The results are shown in Table 1 , which gives the proportion of "old" responses for each of the possible 36 combinations (i.e., identifications of a sentence as one that had appeared previously). First, it can be seen that if a test sentence involves a change in conceptual status, it is characteristically regarded as a "new" sentence. When one goes from normal sentences to strange ones, the proportion of false recognitions averaged over all forms (including same phrasing) is only .17; going from strange sentences to normal ones, the corresponding figure is .13 . Every one of the 18 entries that changes conceptual status, and thus sentence meaning, yields fewer false recognitions than 11 of the 
Table 1

Identification as "Old" Sentence on Incidental Recognition Task (Proportions)

\begin{tabular}{|c|c|c|c|c|c|}
\hline \multirow{2}{*}{\multicolumn{2}{|c|}{$\begin{array}{l}\text { Original Concep- } \\
\text { tual Status } \\
\text { Test Concep- } \\
\text { tual Status }\end{array}$}} & \multirow{3}{*}{$\begin{array}{l}\text { Normal } \\
\text { Normal }\end{array}$} & \multirow{3}{*}{$\begin{array}{l}\text { Perverse } \\
\text { Perverse }\end{array}$} & \multirow{3}{*}{$\begin{array}{l}\text { Normal } \\
\text { Perverse }\end{array}$} & \multirow{3}{*}{$\begin{array}{l}\text { Perverse } \\
\text { Normal }\end{array}$} \\
\hline & & & & & \\
\hline $\begin{array}{l}\text { Original } \\
\text { Phrasing }\end{array}$ & $\begin{array}{c}\text { Test } \\
\text { Phrasing }\end{array}$ & & & & \\
\hline If $p, q$ & $\begin{array}{l}\text { If } p, q \\
p \text { and } q \\
\text { Not } p \text { or } q\end{array}$ & $\begin{array}{l}.74^{*} \\
.77 \\
.52\end{array}$ & $\begin{array}{l}.77^{*} \\
.84 \\
.63\end{array}$ & $\begin{array}{l}.26 \\
.24 \\
.08\end{array}$ & $\begin{array}{l}.16 \\
.16 \\
.14\end{array}$ \\
\hline $\mathrm{p}$ and $\mathrm{q}$ & $\begin{array}{l}\text { If } p, q \\
p \text { and } q \\
\text { Not } p \text { or } q\end{array}$ & $\begin{array}{l}.43 \\
.69 * \\
.40\end{array}$ & $\begin{array}{l}.72 \\
.84 * \\
.18\end{array}$ & $\begin{array}{l}.02 \\
.08 \\
.16\end{array}$ & $\begin{array}{l}.16 \\
.14 \\
.06\end{array}$ \\
\hline $\begin{array}{l}\text { Not } p \\
\text { or } q\end{array}$ & $\begin{array}{l}\text { If } p, q \\
p \text { and } q \\
\text { Not } p \text { or } q\end{array}$ & $\begin{array}{l}.53 \\
.47 \\
.66^{*}\end{array}$ & $\begin{array}{l}.48 \\
.43 \\
.71^{*}\end{array}$ & $\begin{array}{l}.29 \\
.24 \\
.19\end{array}$ & $\begin{array}{l}.08 \\
.10 \\
.14\end{array}$ \\
\hline
\end{tabular}

*Indicates verbatim correct responses; all other responses are errors.

12 nonverbatim cases that maintain conceptual status and meaning (with 13 of the 18 entries that change meaning yielding fewer false recognitions than does that one aberrant case). Next, consider the results for sentences that maintain their original conceptual status. While performance on perverse or strange items is slightly higher than that for normal items, both with regard to verbatim recognitions and "false" recognition of related sentences, basically the overall results are quite similar. When it comes to verbatim recognition, there is not very much difference as a function of phrasing or conceptual status with values ranging from .66 to .84 , with the average proportion of verbatim recognition for normal items .70 and that for strange items .77. Finally, while verbatim recognition is generally better than "false" recognition of related items-there are 12 possible comparisons (i.e., two comparisons for each of six cases), and verbatim recognition is better, with $p<.05$ in 7 of the 12 comparisons-for both normal and strange sentences, there is a considerable amount of "false" recognition for related items, i.e., items semantically more or less equivalent to the originally presented item, but phrased with one of the other sentence operators. On the average, the proportion of such false recognitions is .52 for normal sentences and .55 for strange sentences (which may be compared with the overall average false recognition rate of .15 for sentences that changed conceptual status and thus meaning). The above finding of substantial "false" recognition of sentences semantically equivalent to previously encountered sentences is, of course, the finding of principal interest of the present study.

It appears clear that subjects are sensitive to meaning relations obtaining between warnings phrased as IF, AND, and OR sentences and that sentences that are regarded as meaning equivalent are often confused in an incidental memory task, even though their phrasing involves quite different logical operators, and thus presumably quite different deep structures. These results hold equally well whether the warnings are normal ones or strange, perverse ones. What seems to matter most so far as memory is concerned is not phrasing or conceptual status (as normal or strange), but the fact that a warning involving a particular meaning or propositional content has been encountered.

\section{REFERENCE NOTE}

1. Fillendaum, S. Inducements: On the phrasing and logic of conditional promises, threats, and warnings. Unpublished manuscript, 1974.

\section{REFERENCE}

Fillenbaum, S. Syntactic factors in memory? The Hague, Netherlands: Mouton, 1973.

(Received for publication May 27, 1975.) 